\title{
Upconversion imaging using an all-fiber supercontinuum source
}

Huot, Laurent; Moselund, Peter Morten; Tidemand-Lichtenberg, Peter; Leick, Lasse; Pedersen, Christian

Published in:

Optics Letters

Link to article, DOI:

10.1364/OL.41.002466

Publication date:

2016

Document Version

Peer reviewed version

Link back to DTU Orbit

Citation (APA):

Huot, L., Moselund, P. M., Tidemand-Lichtenberg, P., Leick, L., \& Pedersen, C. (2016). Upconversion imaging using an all-fiber supercontinuum source. Optics Letters, 41(11), 2466-2469.

https://doi.org/10.1364/OL.41.002466

\section{General rights}

Copyright and moral rights for the publications made accessible in the public portal are retained by the authors and/or other copyright owners and it is a condition of accessing publications that users recognise and abide by the legal requirements associated with these rights.

- Users may download and print one copy of any publication from the public portal for the purpose of private study or research.

- You may not further distribute the material or use it for any profit-making activity or commercial gain

- You may freely distribute the URL identifying the publication in the public portal

If you believe that this document breaches copyright please contact us providing details, and we will remove access to the work immediately and investigate your claim. 


\title{
Upconversion imaging using an all-fiber supercontinuum source
}

\author{
Laurent Huot ${ }^{1,2, *}$, Peter Morten Moselund ${ }^{1}$, Peter Tidemand-Lichtenberg ${ }^{2}$, Lasse Leick ${ }^{1}$, \\ and Christian Pedersen ${ }^{2}$ \\ ${ }^{1}$ NKT Photonics A/S, Blokken 84, 3460 Birkerød, Denmark \\ ${ }^{2}$ DTU Fotonik, Frederiksborgvej 399, 4000 Roskilde, Denmark \\ *Corresponding author: Ihu@nktphotonics.com
}

Compiled April 21, 2016

In this paper, the first demonstration of pulsed upconversion imaging using supercontinuum light is presented. A mid-IR imaging system was built by combining a mid-IR supercontinuum source emitting between $1.8 \mu \mathrm{m}$ and $2.6 \mu \mathrm{m}$ with upconversion detection. The infrared signal is used to probe a sample and mixed with a synchronized $1550 \mathrm{~nm}$ laser pulse inside a lithium niobate $\left(\mathrm{LiNbO}_{3}\right)$ crystal. The signal is thus upconverted to the $860 \mathrm{~nm}$ to $970 \mathrm{~nm}$ range and acquired on a standard silicon CCD array at a rate of 22 frames per second. In our implementation, spatial features in the sample plane as small as $55 \mu \mathrm{m}$ could be resolved. () 2016 Optical Society of America

OCIS codes: (110.3080) Infrared imaging; (190.7220) Upconversion; (280.4788) Optical sensing and sensors; (320.6629) Supercontinuum generation

http://dx.doi.org/10.1364/ao.XX.XXXXXX

Throughout the past decade, much attention has been directed toward infrared imaging and infrared spectroscopy [1]. The mid-infrared (mid-IR) spectral region is of particular interest because many chemical compounds have their fundamental absorption bands in this region. Usually, mid-IR detection is done with the help of low band-gap semiconductor materials like indium antimonide ( $\mathrm{InSb}$ ) or mercury cadmium telluride $(\mathrm{HgCdTe})$. Alternatively, microbolometer arrays and thermopiles can also be used. However, these detectors suffer from inherent dark noise and require cooling to perform optimally [2]. Additionally, these detection systems are usually very expensive. The combination of high brightness midIR supercontinuum lasers and upconversion detection systems opens a unique possibility of combining two leading edge technologies pointing towards extremely fast and/or extremely sensitive imaging and spectroscopy in the mid-IR range. Supercontinuum sources are the brightest broadband light sources available today which makes them a powerful tool to analyze a wide range of samples [3]. Upconversion detection circumvents the usual complications of mid-IR detection by translating the midIR signal to the near-infrared (near-IR) wavelength region by nonlinear parametric sum-frequency mixing in a $\chi^{(2)}$ medium. The upconverted signal can thus be detected using an affordable silicon-based CCD array operating in the near-IR region. These detectors are known to work with much better noise and time performance than their counterparts in the mid-IR [4].

In recent years, much research effort has been focused on upconversion detection both for imaging and spectroscopy using either bulk or waveguide structures in the nonlinear material. Pulsed upconversion has been demonstrated and successfully used for spectroscopy applications [5]. Broadband illumination in an intracavity upconversion setup has been shown to enhance the angular field of view [6]. Also, hyperspectral image reconstruction has been achieved using an intracavity upconversion imaging system in conjunction with a broadband thermal source [7]. Lastly, upconversion using a supercontinuum source has been reported in an intracavity upconversion scheme using the high circulating power of a continuous wave pump laser for spectroscopy [8].

In this paper, we rely on the high brightness of an IR supercontinuum source to build what to our knowledge is the first single-pass pulsed upconversion imaging system based on supercontinuum. This system has the special feature of relying on the same pump laser to synchronously generate both the broadband signal pulse and the pump pulse for the nonlinear mixing process. This both eliminates the need for a second laser and ensures perfect temporal overlap of the supercontinuum and conversion pump pulses. However, it would in the future be of high interest to investigate the possibility of using two separate electronically synchronized lasers to generate the supercontinuum and the conversion pump as this enables delay tuning between the two signals which might hold strong potential in remote sensing applications like LIDAR and hyperspectral imaging.

The high peak power of the conversion pump pulses ensures the upconversion of the IR signal and suppresses the need for an intracavity setup. A single-pass setup with synchronous pumping [5] relaxes the demanding constraints for the nonlinear material to have high nonlinearity and low transmission losses. This allows the use of bulk crystals which can easily be manufactured in sizes of the order of several $\mathrm{mm}$, which allows for the use of a large beam diameter in the nonlinear crystal thus increasing spatial and spectral resolution. Secondly, super- 


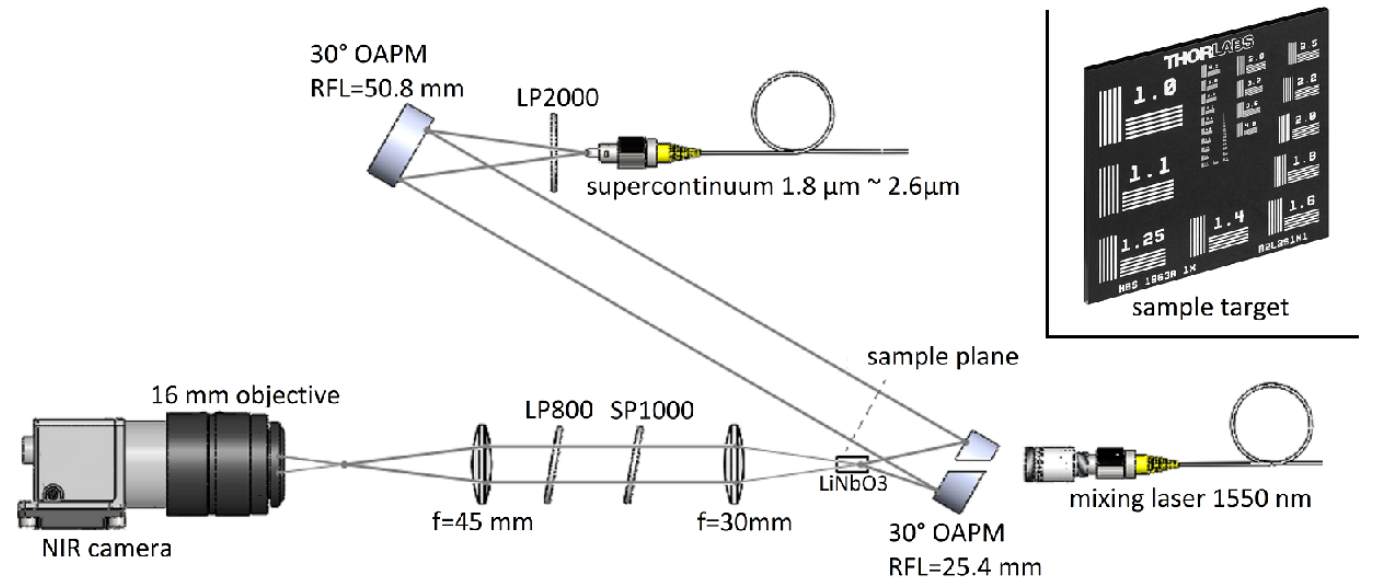

Fig. 1. Top view schematic representation of the pulsed upconversion imaging setup. The use of OAPMs make for a mostly achromatic setup. The broadband signal pulse is combined with the $1550 \mathrm{~nm}$ pump pulse through a hole drilled through an OAPM.

continuum sources further in the IR spectrum may be upconverted using a similar scheme exploiting crystals like $\mathrm{AgGaS}_{2}$ or $\mathrm{AgGaSe}_{2}$ [9].

The pulsed nature of the mixing laser will improve the signal to noise ratio of the system by eliminating the upconversion of background between signal pulses. As the duty cycle of the laser is approximately 1:10000 the pulsed upconversion can reduce the impact of upconverted background light by up to 40 $\mathrm{dB}$.

In our experiment, the source relies on a $1550 \mathrm{~nm}$ erbium fiber-laser delivering $3.5 \mathrm{~ns}$ pulses at $40 \mathrm{kHz}$ as pump source. The pulse is then separated into two arms with a 90/10 fiber coupler. The higher power pulse is made to propagate through a combination of standard single-mode silica fibers and a section of passive thulium doped fiber used to generate a supercontinuum ranging from $1.8 \mu \mathrm{m}$ to $2.6 \mu \mathrm{m}$ with an average power of approximately $200 \mathrm{~mW}$ [3]. The lower power pulse is propagated through a standard single-mode silica fiber of the same length as the high power arm so that the two pulses will be synchronized at the outputs of the source. The $1550 \mathrm{~nm}$ pulse will be used as the mixing pump in the upconversion process. The raw spectrum of the supercontinuum can be seen in Fig. 2. It is of interest to note the presence of a strong peak at $2.38 \mu \mathrm{m}$ which in the following measurements will show up as a bright ring in the images.

The synchronized supercontinuum and mixing pulses are delivered to the setup through two separate fibers as indicated in Fig. 1. Both the supercontinuum and the mixing laser are randomly polarized. The supercontinuum light is first filtered with a $2000 \mathrm{~nm}$ long-pass filter. This filtering is necessary because for the crystal cut angles used in the experiment, the shorter wavelengths of the supercontinuum could get upconverted by self mixing with the other wavelength components of the supercontinuum thus creating parasitic light in the image place. After this filter, the supercontinuum spans from 1950 $\mathrm{nm}$ to $2600 \mathrm{~nm}$. The supercontinuum is then collimated to a $12 \mathrm{~mm}$ diameter beam using a $50.8 \mathrm{~mm}$ reflected focal length $30^{\circ}$ off axis parabolic mirror (OAPM) and is shone through the sample plane containing a US Air Force 1963 resolution test target. The sample plane is transformed by a $25.4 \mathrm{~mm}$ focal length $30^{\circ} \mathrm{OAPM}$ to create a two-dimensional Fourier transform of the sample's transmission in the nonlinear crystal [10]. This plane

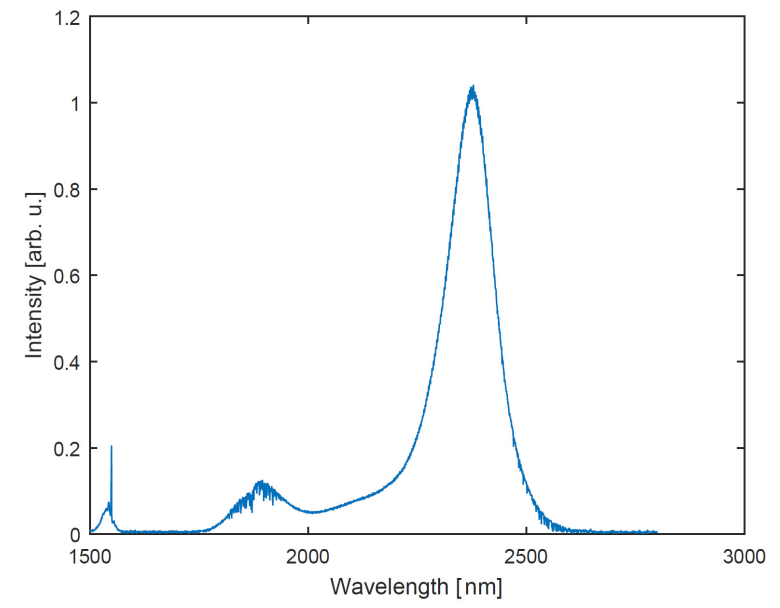

Fig. 2. Spectrum of the supercontinuum. It can be noted that the strong peak at $2.38 \mu \mathrm{m}$ provides a recognizable spectral signature that will be used to investigate the distribution of wavelengths across the image plane.

will be referred to as the Fourier plane. It is of interest to note that the use of OAPMs allows for an achromatic system, capable of operating with good performance accross a very wide spectral band. This is particularly relevant in the infrared where designing reflection and transmission coatings operating across wide spectral bands can become a major challenge. It is important to choose mirrors with the smallest possible reflection angles. Indeed, while OAPMs offer excellent collimation, and point-focusing performances, the imaging process is hindered by the fact that the focal length of each sub-area of the mirror is different, resulting in lateral field distortion when used for imaging applications. Using parabolic mirrors designed for incidence angles closer to normal incidence reduces this effect. The pump arm is collimated to a $2.2 \mathrm{~mm}$ diameter beam and is shone through a $3 \mathrm{~mm}$ diameter hole drilled at the center of the OAPM. The beams are superimposed inside a $5 \mathrm{~mm} \times 5 \mathrm{~mm}$ $x 10 \mathrm{~mm} \mathrm{LiNbO} 3$ crystal cut at $48^{\circ}$. The crystal is placed on a rotation stage to allow for angle tuning of the phase-matching condition. The sum-frequency generation process relies on type 


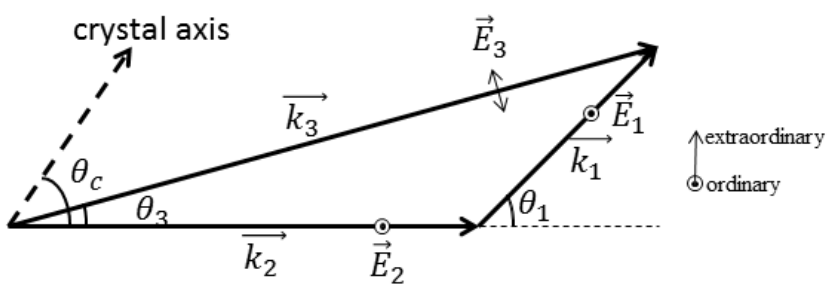

Fig. 3. Type I phase-matching upconversion scheme. The two input beams are polarized along the crystals ordinary axis and the upconverted signal is polarized along the extraordinary direction. $\overrightarrow{k_{1}}, \overrightarrow{k_{2}}$ and $\overrightarrow{k_{3}}$ represent the wavevectors of the input signal, pump, and upconverted signal respectively.

I phase-matching. The phase-matching scheme is illustrated in Fig. 3. As a result of the nonlinear process, the IR signal gets converted to the $860 \mathrm{~nm}$ to $970 \mathrm{~nm}$ range [11]. The IR signal, pump and upconverted signal wavelengths $\lambda_{1}, \lambda_{2}$ and $\lambda_{3}$ respectively are linked by the relation stated in Eq. 1 .

$$
\frac{1}{\lambda_{3}}=\frac{1}{\lambda_{1}}+\frac{1}{\lambda_{2}}
$$

The relation between the mixing angles and the wavelengths is given by Eq. 2 .

$$
k_{1}^{2}=k_{2}^{2}+k_{3}^{2}-2 k_{2} k_{3} \cos \theta_{3}
$$

In this equation $k_{1}, k_{2}$ and $k_{3}$ represent the wavenumbers of wavevectors $\overrightarrow{k_{1}}, \overrightarrow{k_{2}}$ and $\overrightarrow{k_{3}}$ which correspond to the input signal, the pump, and the upconverted signal respectively.

The near-IR plane waves exciting the crystal are then propagated through a simple Keplerian beam expander. The first lens is a $30 \mathrm{~mm}$ focal length plano-convex placed at $30 \mathrm{~mm}$ of the Fourier plane. The second lens is a $45 \mathrm{~mm}$ focal length planoconvex lens. The near-IR signal is imaged on an affordable nearIR CCD camera equipped with a $16 \mathrm{~mm}$ focal length objective lens. This last lens has its object focal plane coinciding with the image plane out of the beam expander, and thus performs the inverse Fourier transform of the near-IR signal and restitutes the spatial information of the sample object.

The quantum efficiency of the phase-matched light traveling along the center of the nonlinear crystal is calculated to be $1.2 * 10^{-5}$ [11]. The $2.2 \mathrm{~mm}$ diameter of the pump beam is here the main parameter reducing the efficiency. Additionally, the setup does not implement any polarization control which causes a decrease in efficiency of a factor four. However, the high number of available IR signal photons allows the easy detection of an image on the CCD camera. It can be noted that other crystals might offer more efficient upconversion. Silver gallium sulfide $\left(\mathrm{AgGaS}_{2}\right)$ in particular has an effective nonlinear susceptibility of $16 \mathrm{pm} / \mathrm{V}$ for this process which would translate to an order of magnitude increase in efficiency compared to $\mathrm{LiNbO}_{3}$ the effective nonlinearity of which is -4.1 $\mathrm{pm} / \mathrm{V}$. First, images were made without the resolution target. As can be seen in Fig. 4, a consequence of the angular dependent phase-matching condition seen from Eq. 2 is that different spectral components of the object light are upconverted at different angles. A bright spectral component in the object light will result in a bright ring in the image. The phase-matching condition is highly dependent on the cut angle, rotation and temperature of the crystal. This is the property that can be exploited to reconstruct hyperspectral images by angle or temperature tuning of the crystal or by translating the sample [7].

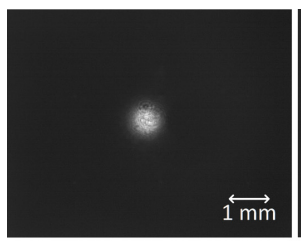

(a)

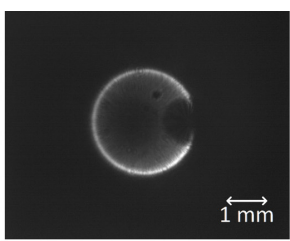

(b)

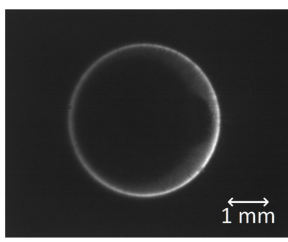

(c)

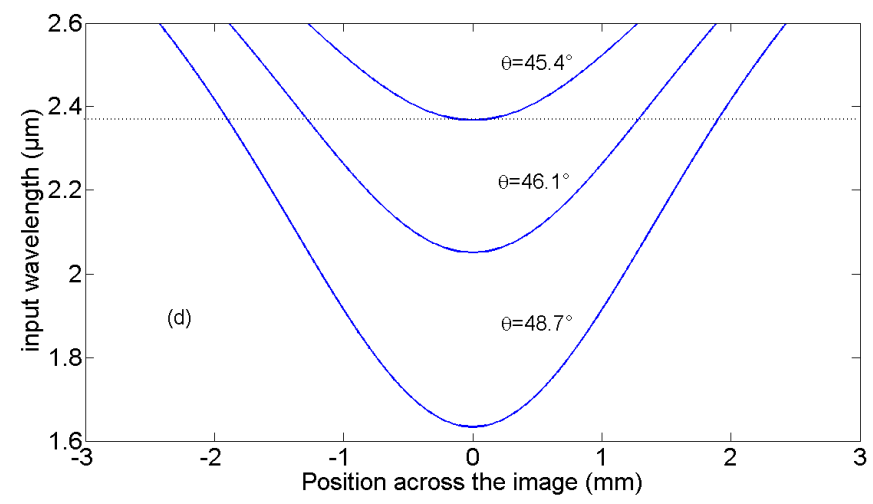

Fig. 4. (a), (b) and (c) are acquired images of upconverted image of object light when no sample is present for different angular positions of the crystal corresponding to internal angles of $\theta_{c}=45.4^{\circ}, \theta_{c}=46.1^{\circ}$ and $\theta_{c}=48.7^{\circ}$ respectively. The radii of the bright rings are measured to be respectively 0.1 $\mathrm{mm}, 1.3 \mathrm{~mm}$ and $1.9 \mathrm{~mm}$. Figure (d) displays the theoretical distribution of wavelengths in the image plane. A dotted line marks the intensity peak of the supercontinuum. Additionally, the dark circle that can be observed on the right hand side in figures (b) and (c) is due to the hole in the OAPM.

We note that the spatial distribution of spectral components in the upconverted image is in good agreement with the emission spectrum of the supercontinuum source. Figures 4(a)-4(c) show the acquired upconverted images in the case where there is nothing in the sample plane for three different angular positions of the crystal. The bright peak at $2.38 \mu \mathrm{m}$ in the spectrum of the supercontinuum directly translates to a bright ring in the upconverted image with a radial position which depends on the crystal rotation. The lower spectral brightness region of the supercontinuum spectrum is upconverted as a darker area mostly towards the center of the image. Figure 4.(d) shows the theoretical distribution of wavelengths across a diameter of the image. The horizontal dotted line represents the peak at 2.38 $\mu \mathrm{m}$ of the spectrum of the supercontinuum. For the three different angles, the abscissa of the intersections of this line with the curves gives us the theoretical radii of the bright rings in the upconverted images. These correspond to the measured diameters of the acquired upconverted images.

To further investigate the correlation between the spatial distribution of wavelengths in the upconverted image and the theoretical distribution anticipated by Eq. 2, a fiber-coupled spectrometer was used to probe the spectral content across the image plane along a diameter of the image. The experimental values are confronted with the theoretical curve in Fig. 5. We can observe good correlation between the experimental and theoretical data. The fact that we can easily link the position on the detector to the corresponding infrared object wavelength make this source suitable for spectroscopy, and hyperspectral imag- 


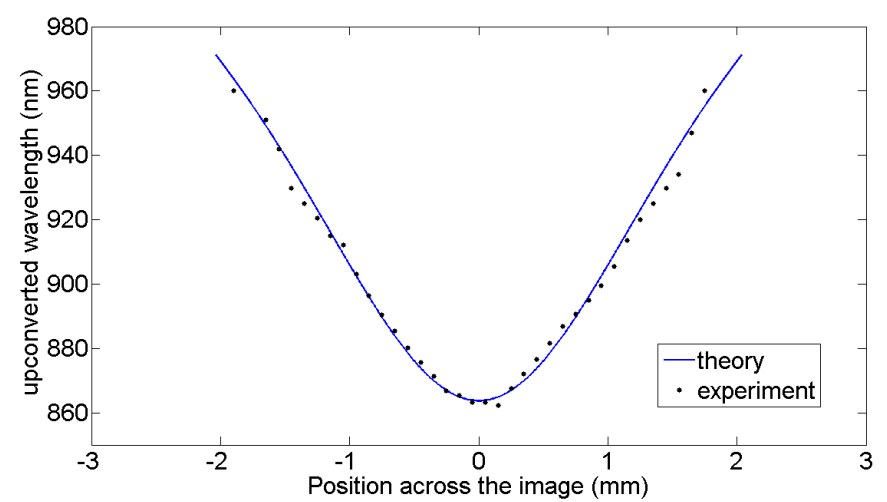

Fig. 5. Spectral components across a diameter of the image for an angular position of the crystal corresponding to an internal angle of $\theta_{c}=46.5^{\circ}$. The dotted line represents the experimental data and the full line represents the theoretical values.

ing applications.

A US Air Force 1963 resolution target is placed in the sample plane. Figure 6 and visualization 1 shows upconverted images that were acquired in real time at a rate of 22 frames per second. We can observe some lateral field distortion which is believed to be due to the use of OAPMs as discussed above. Additionally, the brighter outer ring due to the peak at $2.38 \mu \mathrm{m}$ in the spectrum of the supercontinuum is still present in these upconverted images. The image formed is $4.2 \mathrm{~mm}$ in diameter and the imaging resolution was measured as the smallest resolvable line of the resolution target. Resolutions down to $55 \mu \mathrm{m}$ were measured in the center of the image. We notice that the vertical lines of the resolution target appear blurred when compared to the horizontal lines. The $\mathrm{LiNbO}_{3}$ crystal being placed at a slight angle with respect to the pump beam, multiple reflections of the input beams inside the $\mathrm{LiNbO}_{3}$ crystal would result in multiple images being formed with a slight horizontal offset from one another thus causing the image to be blurred along one direction. Alternatively, we suspect that the aberrations introduced by the OAPMs could result in the vertical lines appearing more blurry than the horizontal lines.

In conclusion, we have demonstrated the first pulsed upconversion imaging system using a supercontinuum light source. A complex infrared signal in the $2 \mu \mathrm{m}$ to $2.6 \mu \mathrm{m}$ range was thus successfully upconverted to the $860 \mathrm{~nm}$ to $970 \mathrm{~nm}$ range and acquired on an affordable near-IR CCD camera. Additionally, The use of the high brightness supercontinuum and the synchronous pulsed upconversion scheme drastically expands the choice of usable crystals. In this case, the use of a large pump beam diameter inside a bulk $\mathrm{LiNbO}_{3}$ crystal has allowed us to obtain images with good spatial resolution.

As a consequence of the phase-matching condition, spectral components are upconverted in different parts of the image, which makes this system an interesting choice for spectroscopy and hyperspectral imaging applications.The fast acquisition time would make it suitable for on line industrial vision applications, fast gas sensing and bio-medical imaging. Finally, this particular system has the advantage of being mostly achromatic. By choosing the nonlinear crystal appropriately, we can rely on a very similar system to perform pulsed upconversion imaging further in the infrared using novel long wavelength supercontinuum sources even further in the infrared.

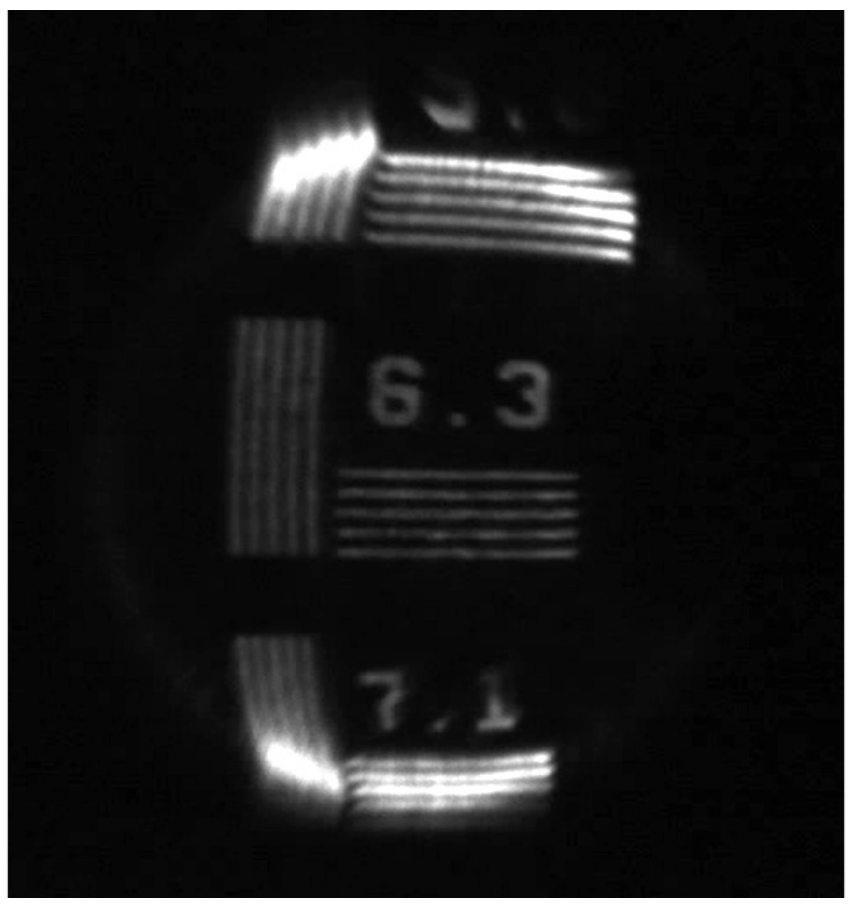

Fig. 6. Acquired upconverted image of a part of an US Air Force resolution test target. The angle between the crystal axis and the pump beam is $46.5^{\circ}$. Images were acquired in real-time at a rate of 22 images per second. A real time video acquisition is provided in Visualization 1.

\section{FUNDING}

Mid-TECH - H2020-MSCA-ITN-2014 Grant agreement no: 642661

\section{REFERENCES}

1. J. S. Dam, P. Tidemand-Lichtenberg, and C. Pedersen, Nat Photonics 6, 788 (2012).

2. Infrared Detectors (CRC Press, 2011).

3. V. V. Alexander, O. P. Kulkarni, M. Kumar, C. Xia, M. N. Islam, F. L. T. Jr., M. J. Welsh, K. Ke, M. J. Freeman, M. Neelakandan, and A. Chan, Optical Fiber Technology 18, 349 (2012).

4. L. Høgstedt, J. S. Dam, A.-L. Sahlberg, Z. Li, M. Aldén, C. Pedersen, and P. Tidemand-Lichtenberg, Opt. Lett. 39, 5321 (2014).

5. S. Dupont, P. M. Moselund, L. Leick, J. Ramsay, and S. R. Keiding, J. Opt. Soc. Am. B 30, 2570 (2013).

6. A. J. Torregrosa, H. Maestre, and J. Capmany, Opt. Lett. 40, 5315 (2015).

7. L. M. Kehlet, N. Sanders, P. Tidemand-Lichtenberg, J. S. Dam, and C. Pedersen, Opt. Express 23, 34023 (2015).

8. P. Moselund, C. Petersen, L. Leick, J. S. Dam, P. Tidemand-Lichtenberg, and C. Pedersen, "Highly stable, all-fiber, high power zblan supercontinuum source reaching $4.75 \mu \mathrm{m}$ used for nanosecond mid-ir spectroscopy," in "Advanced Solid-State Lasers Congress," (Optical Society of America, 2013), p. JTh5A.9.

9. D. A. Roberts, Appl. Opt. 35, 4677 (1996).

10. J. E. Midwinter, Applied physics letters 12 (1968).

11. Nonlinear Optics third edition (Elsevier, 2008). 


\section{FULL REFERENCES}

1. J. S. Dam, P. Tidemand-Lichtenberg, and C. Pedersen, "Room temperature mid-ir single photon spectral imaging," Nat Photonics 6, 788-793 (2012).

2. Infrared Detectors (CRC Press, 2011).

3. V. V. Alexander, O. P. Kulkarni, M. Kumar, C. Xia, M. N. Islam, F. L. T. Jr., M. J. Welsh, K. Ke, M. J. Freeman, M. Neelakandan, and A. Chan, "Modulation instability initiated high power all-fiber supercontinuum lasers and their applications," Optical Fiber Technology 18, 349-374 (2012).

4. L. Høgstedt, J. S. Dam, A.-L. Sahlberg, Z. Li, M. Aldén, C. Pedersen, and P. Tidemand-Lichtenberg, "Low-noise mid-ir upconversion detector for improved ir-degenerate four-wave mixing gas sensing," Opt. Lett. 39, 5321-5324 (2014).

5. S. Dupont, P. M. Moselund, L. Leick, J. Ramsay, and S. R. Keiding, "Up-conversion of a megahertz mid-ir supercontinuum," J. Opt. Soc. Am. B 30, 2570-2575 (2013).

6. A. J. Torregrosa, H. Maestre, and J. Capmany, "Intra-cavity upconversion to $631 \& \# \times 2009 ; \& \# \times 2009$;nm of images illuminated by an eye-safe ase source at 1550\&\#x2009;\&\#x2009;nm," Opt. Lett. 40, 5315-5318 (2015).

7. L. M. Kehlet, N. Sanders, P. Tidemand-Lichtenberg, J. S. Dam, and C. Pedersen, "Infrared hyperspectral upconversion imaging using spatial object translation," Opt. Express 23, 34023-34028 (2015).

8. P. Moselund, C. Petersen, L. Leick, J. S. Dam, P. TidemandLichtenberg, and C. Pedersen, "Highly stable, all-fiber, high power zblan supercontinuum source reaching $4.75 \mu \mathrm{m}$ used for nanosecond mid-ir spectroscopy," in "Advanced Solid-State Lasers Congress," (Optical Society of America, 2013), p. JTh5A.9.

9. D. A. Roberts, "Dispersion equations for nonlinear optical crystals: Kdp,aggase2, and aggas2," Appl. Opt. 35, 4677-4688 (1996).

10. J. E. Midwinter, "image conversion from $1.6 \mu \mathrm{m}$ to the visible in lithium niobate," Applied physics letters 12 (1968).

11. Nonlinear Optics third edition (Elsevier, 2008). 